\title{
TTR
}

Traduction, terminologie, re?daction

\section{Plurilinguisme et traduction - Le vernaculaire noir américain : enjeux, réalité, réception à propos de The Sound and the Fury}

\section{Bernard Vidal}

Volume 4, numéro 2, 2e semestre 1991

Traduire la théorie

URI : https://id.erudit.org/iderudit/037097ar

DOI : https://doi.org/10.7202/037097ar

Aller au sommaire du numéro

Éditeur(s)

Association canadienne de traductologie

ISSN

0835-8443 (imprimé)

1708-2188 (numérique)

Découvrir la revue

Citer cet article

Vidal, B. (1991). Plurilinguisme et traduction - Le vernaculaire noir américain : enjeux, réalité, réception à propos de The Sound and the Fury. TTR, 4(2),

151-188. https://doi.org/10.7202/037097ar d'utilisation que vous pouvez consulter en ligne. 


\section{Plurilinguisme et traduction - Le vernaculaire noir américain: enjeux, réalité, réception à propos de The Sound and the Fury ${ }^{1}$}

\section{Bernard Vidal}

"Le roman est une expansion et un approfondissement de l'horizon linguistique, un affinement de notre perception des differenciations socio-linguistiques. ${ }^{2}$

1. Plurilinguisme et traduction: mise en place théorique

En 1929-30, Bakhtine, réagissant contre ce qu'il appelait «l'objectivisme abstrait» critiquait une vision du langage dégagée de toute contrainte existentielle où, selon lui, «on isole arbitrairement, on objectifie, réifie le concept de langue, on en fait [...] un système de nature abstraite et conventionnelle». $\grave{A}$ ses yeux au contraire, le langage, fait social par

1. Dans cet article, l'auteur reprend la problématique de sa thèse de doctorat.

2. Mikhail Bakhtine, Esthétique et théorie du roman (Moscou, 1975). Traduit du russe par Daria Olivier, préface de Michel Aucouturier (Paris, NRF Gallimard, 1978), p. 182. 
excellence, se construit par la pratique et l'énonciation ${ }^{3}$, et aucun énoncé ne saurait exister «in vitro» ou «in vacuum». En outre, l'acte de parole individuel, étant toujours orienté vers autrui, sera le lieu d'une interaction incessante entre les deux pôles de la communication, celui d'une intersubjectivité entre le moi et l'autre.

Cette bivocalité dynamique, ou dialogisme, pierre angulaire sur laquelle repose tout l'édifice de la pensée bakhtinienne, empêche une vision unitaire, individuelle, logocentrique et centripète des faits de langue autant que de société. Un tel système présuppose nécessairement une vision autre du monde: l'humain sera avant tout un inter-humain, et l'existence, une co-existence où rien n'existe par soi-même, en isolement absolu. Dans ces conditions, le moi, traversé de part en part d'un foisonnement de langages et de voix, ne peut se concevoir que comme le jeu (conflictuel?) de leurs multiples résonnances idéologiques.

Il en sera de même en littérature où le style romanesque se définira plus par son aspect social qu'individuel. On ne saurait avoir affaire seulement à «un verbe linguistique abstrait au service de la maîtrise d'un artiste». Bakhtine est on ne peut plus clair à ce propos: «Le postulat de la véritable prose romanesque, c'est la stratification interne du langage, la diversité des langages sociaux et la divergence des voix individuelles qui y résonnent»" Pas question donc de méconnaître «la vie sociale du verbe hors de l'atelier de l'artiste, dans les vastes espaces des places publiques, des rues, des villes et villages, des groupes sociaux, des générations et des époques». Le principe dialogique oblige à l'ouverture, à la conscience multilingue et polyphonique. Celle-ci entraîne en retour une relativisation et un

3. Mikhail Bakhtine, le Marxisme et la philosophie du langage. Première édition sous le nom de Volochinov (Leningrad, 1929). Traduction et présentation de Marina Yaguello (Paris, Éditions de Minuit, 1977), pp. 93-97.

4. Bakhtine, 1978, p. 90.

5. Ibid., p. 85 . 
décentrement de l'activité littéraire ${ }^{6}$. Le plurilinguisme, la plurivocalité, l'hétéroglossie sont autant de nouveaux concepts par rapport auxquels tout créateur d'un univers romanesque ne peut manquer de situer son cuvre, sciemment ou non.

La conscience linguistique socio-idéologique concrète, en devenant activement créatrice, c'est-à-dire littérairement active, se découvre par avance environnée de plurilinguisme et nullement par un langage unique indiscutable et péremptoire. Toujours et partout, à tous les âges de la littérature historiquement connus, la conscience littérairement active découvre des langages et non un langage. Elle se trouve devant la nécessité $d u$ choix d'un langage. Dans chacune de ses manifestations littéraires verbales, elle s'oriente activement parmi le polylinguisme, y occupe une position, choisit, un «langage». Ce n'est qu'en demeurant dans une existence fermée, sans écriture, ni pensées, à l'écart de toutes les voies du devenir socio-idéologique, que l'homme ne perçoit pas cette activité 'inguistique élective et peut rester tranquillement dans la certitude absolue et la prédétermination de sa langue à lui? .

Bakhtine synthétise ici de façon remarquable la problématique, essentielle à notre propos, qui confronte le démiurge littéraire. Quiconque pratique la littérature sait qu'il doit se situer dans le fourmillement des langages humains: langages de la rue et des salons, de la nation et des régions, de la famille et de la société, de l'école et de la profession, etc., surtout au sein d'un idiome apparemment unique $^{8}$. L'écrivain «s'oriente parmi le polylinguisme» inhérent à tout

6. Ibid. p. 186.

7. Ibid., p. 116. Les italiques sont de Bakhtine.

8. Les littératures nationales ont réagi bien différemment devant cette problématique et il y aurait là une étude intéressante à accomplir entre les deux littératures qui nous intéressent. Sans trop vouloir nous avancer dans des généralités compromettantes, il nous semble néanmoins possible de dire que le roman américain a très tôt fait preuve d'une remarquable conscience sociolectale plurilingue, ce qui 
système, libre d'assumer la diversité langagière du monde qui l'entoure, et donc de l'inscrire dans son cuvre, ou, au contraire, de la rejeter, d'être sourd à ses échos et, par voie de plume, de la nier à ses personnages dont il inféode alors la parole à son monolinguisme, «du haut de l'indiscutable autorité d'un langage unique ${ }^{9}$. Une technique romanesque renvoie toujours à la métaphysique du romancier énoncera Sartre quelques années plus tard ${ }^{10}$. Bakhtine ne disait pas autre chose. La position assumée par le narrateur face au polylinguisme de son temps constituera bel et bien, au même titre que l'«objectivité» ou l' «omniscience», un informant de sa vision idéologisée de son univers.

Introduit dans le roman, le plurilinguisme y est soumis à une élaboration littéraire. Les voix sociales et historiques qui peuplent le langage (tous ses mots, toutes ses formes), qui lui donnent des significations concrètes, précises, s'organisent dans

ne fut pas nécessairement le cas de son homologue français. Que l'on songe, dès 1851, à l'cuvre de Melville, ou même, au classique de Harriet Beecher Stowe, Uncle Tom's Cabin. L'auteure nous y présente, cela en 1852, un remarquable panorama sociolectal de sa société. N'oublions pas non plus les romans de William Dean Howells publiés dans la deuxième moitié du siècle et écrits par un romancier passionné de faits de langue. Un autre des représentants de cette tendance sera Dos Passos, que nous mentionnerons à l'occasion dans notre étude. Son ouvrage Manhattan Transfer pourrait être tenu comme une sorte de culmination du plurilinguisme. Pour ce qui est des enjeux sociolectaux dans la littérature américaine on pourra se reporter à l'ouvrage de Elsa Nettels Language, Race, and Social Class in Howells's America (Lexington, The University Press of Kentucky, 1988); ainsi qu'à celui de Kenneth Cmiel, Democratic Eloquence, The Fight Over Popular Speech in Nineteenth Century America (New York, William Morrow and Company, 1990).

9. Bakhtine, op. cit., 1978, p. 184.

10. D'ailleurs, fort opportunément, c'est à propos du Bruit et la fureur que Sartre dans son article de juillet 1939 formulait cette opinion. Bien qu'elle fût motivée par un enjeu autre que le polylinguisme ( $\ll \mathrm{La}$ temporalité chez Faulkner» pour être plus précis), elle n'en conserve pas moins toute sa validité face au problème qui nous intéresse. 
le roman en un harmonieux système stylistique, traduisant la position socio-idéologique différenciée de l'auteur au sein du plurilinguisme de son époque ${ }^{11}$.

Si, à l'heure actuelle, la plupart des concepts bakhtiniens relatifs à la plurivocalité semblent avoir acquis droit de cité dans le vocabulaire des critiques contemporains, leur entrée demeure encore bien modeste dans celui des traducteurs ${ }^{12}$. Pourtant, doit-on rester indifférent aux enjeux que soulève la plurivocalité du texte source? Comment en outre saisir son intertextualite ${ }^{13}$ ? Ou, comme nous allons

11. Bakhtine, op. cit., 1978, p. 121. C'est nous qui soulignons. L'italique est de Bakhtine.

12. Toutefois, un certain nombre d'ouvrages récents marquent l'ouverture des études en traductologie à l'influence bakhtinienne. 'D'une part, celui d'Annie Brisset, Sociocritique de la traduction, Théâtre et altérité au Québec (1968-1988) paru aux Éditions du Préambule en 1990, puisque la traduction y est vue comme une énonciation soumise au discours social de son temps, donc aux configurations idéologiques. Puis, plus récemment, celui de Douglas Robinson The Translator's Turn (Baltimore and London, Johns Hopkins University Press, 1991). Partant du dialogisme bakhtinien (entre autres) et de l'aspect inéluctablement idéologique du langage, $D$. Robinson se fait l'avocat d'une pratique «somatique» (c'est-à-dire résolument personnalisée) de la traduction. Mentionnons aussi Discourse and the Translator dont nous parlons à la note suivante.

13. On ne s'étonnera pas que de toutes les notions héritées de Bakhtine directement - ou indirectement, via Kristeva, dans ce cas-ci intertextualité soit celle qui ait le plus facilement migré vers les études en traductologie. Todorov proposait intertextualité comme terme de remplacement à dialogisme, qu'il jugeait embarrassé d'une multitude d'acceptions. Le même reproche pourrait être adressé aujourd'hui à intertextualité qui pose bien des problèmes à la conscience critique et, conséquemment, à celle du traducteur qui choisirait d'y être sensible. Le premier et non le moindre étant celui de la validité opératoire du terme. Marc Angenot nous semblait avoir fait le tour de la question et son article de 1983 (Revue des sciences humaines, tome $\mathrm{LX}, \mathrm{n}^{\circ} 189$ ) résumait très bien à nos yeux la problématique du mot dont l'ambigüté notionnelle n'a pas empêché 
le voir, peut-on demeurer sourd à son plurilinguisme ${ }^{14}$ ? Ces questions, une certaine vision réductrice de la traduction ne peut les prendre en compte, coincée où elle se trouve entre, d'une part, un a priori d'intemporalité toujours vivace et, d'autre part, l'aporie d'un dualisme qu'elle postule irréconciliable entre le fond et la forme, entre le système et le discours - héritière en cela d'une linguistique structurale indifférente à l'aspect idéologique du langage. Elle ne peut ainsi mesurer tout le profit d'une pensée qui, quoique fondée sur une bipolarité, enseigne néanmoins à ne pas l'appliquer comme une exclusion (l'un ou/contre l'autre) mais comme une dyade ou, mieux encore, comme une simultanéite (l'un ou/en même temps que l'autre). Comment, selon cette pensée, le fond jouerait-il contre la forme? Les deux s'exigent mutuellement, aucun n'étant en soi-même suffisant, et entretiennent par ailleurs entre eux un rapport hautement sémiotisé.

Si maintenant nous comparons nos textes d'arrivée avec leurs originaux, nous constaterons que l'alternative initiale, monologisme ou plurilinguisme, n'a pas été acceptée de la même manière par le traducteur que par le créateur, et que le passage d'une langue à l'autre s'est accompagné d'un passage du plurilinguisme au monologisme. Apparaît ici un problème fondamental au traduire qui est loin d'être unique aux textes que nous étudions : celui de la traduction des langages sociaux - ou plutôt, celui de la méconnaissance de la littérature orale. Attitude suffisamment répandue pour que Berman y voie trois des

la diffusion dans le monde de la traduction. Ainsi, dans leur ouvrage Discourse and the Translator (London and New York, Longman, 1990), B. Hatim et I. Mason consacrent un chapitre entier à l'intertextualité: Intertextuality and intentionality, pp. 120-137. Toutefois, le sens qu'ils lui donnent ne se démarque pas suffisamment de celui, plus banal, d'influence et d'allusion. Continue-t-on, ainsi que le remarquait Marc Angenot, à faire du neuf avec du vieux?

14. Ce terme appelle un éclaircissement en particulier après le travail d'Annie Brisset sur le discours social et la traduction. Notre conception du mot écarte la présence de celui d'interdiscursivité ou de discours social pour se réduire à un contenu plus banal de multiplicité des langues ou sociolectes en présence. Bakhtine luimême n'établit pas toujours une frontière nette entre les deux acceptions. Nous tenons à les dissocier. 
douze tendances déformantes de la traduction (10. La destruction des réseaux vernaculaires et leur exotisation; 11 . La destruction des locutions et idiotismes; 12. L'effacement des superpositions de langue $)^{15}$.

Dos Passos choisissant de peindre Manhattan dans son foisonnement existentiel aurait pu s'en tenir à la seule peinture du phénomène d'un point de vue narratif traditionnel. Sa description se serait déroulée selon l'ordre spatio-temporel logique et ses personnages, figés dans un discours d'escorte englobant, se seraient exprimés selon l'usage dominant de l'époque. Tel ne fut pas le cas, nous le savons, et l'écrivain a résolument opté pour le polylinguisme, façonnant son cuvre dans l'art d'une mosaïque tant verbale que spatiale. À l'unisson, Faulkner aurait pu adhérer sa carrière durant au crédo naturaliste le plus conventionnel et il ne nous aurait pas légué les cuvres que nous connaissons. Or, là encore, nous savons quel sort il a réservé par ses choix techniques, au temps, dans The Sound and the Fury, à la conscience monologique dans As I Lay Dying, et au discours dans Light in August ${ }^{16}$. L'un comme l'autre a donc opté pour des procédés narratifs bien précis. N'est-il pas navrant dès lors de constater que ces choix, pourtant essentiels, n'ont pas été assumés avec la même rigueur par les traducteurs? Qu'une contrainte aussi fondamentale que celle de la répartition des scènes dans Manhattan Transfer n'est pas scrupuleusement respectée; que le carnavalesque faulknérien s'est mué en tragique; que le polylinguisme initial s'est estompé. Le travail du traducteur a privilégié de la sorte une vision idéalisée du fond au détriment de la forme, et amputé l'œuvre d'une moitié de sa poétique, oubliant ou ne sachant pas «qu'un texte est le sens de ses formes autant

15. Antoine Berman, Texte, Traduction/Textualite, $\mathrm{n}^{\circ} 4,1985$, p. 80-81.

16. Nous profitons de l'énumération de ces titres pour spécifier que, à nos yeux, la mention du titre en anglais ou en français n'est pas indifférente. L'enjeu même de notre travail nous force à établir une distinction (une séparation?) entre le titre anglais (écrit par Dos Passos, Faulkner) et le titre français, résultat du travail du traducteur, Coindreau. 
que le sens de ses mots». ${ }^{17}$ La création étrangère, considérablement aseptisée, perd un volet de son aspect novateur et une bonne partie de sa vigueur. Mais, ce qui est plus grave, tous les effets de sens inhérents à la répartition du discours, tous les enjeux de la mimésis verbale sont de la sorte escamotés. Dos Passos francisé n'offre qu'une seule teinte langagière lui qui, révolutionnaire, donnait la parole à de multiples accents, groupes ou ethnies. La traduction aboutit alors à une pratique qui refuse la re-création et concrétise cette distinction entre créateur et traducteur que dénonçait Meschonnic.

L'opposition entre créateur et traducteur semble généralement admise. Sa force idéologique est telle aujourd'hui qu'il est sans doute bien difficile de ruiner l'erreur, de la montrer d'abord, même. Comme entre créateur et critique, créateur et lecteur. Elle est liée, pour la traduction, à un impérialisme culturel arrivé, qui a oublié son histoire et n'aime pas à se la faire rappeler. Elle est liée à une sacralisation de la littérature qui comporte aussi sa permanente mise hors service. Son esthétisation la désarme. La critique joue le jeu. L'enseignement le perpétue. Il tente de se rattraper par une teinte de sociologie ou un peu de psychanalyse. L'empirisme des traducteurs et de la plupart des «théoriciens», situé dans l'idéologie traditionnelle de la littérature, constate que la plupart des traducteurs ne sont pas des «créateurs» ${ }^{18}$.

Dans ces conditions, comment peut-on attendre du public de la langue d'arrivée qu'il apprécie au maximum de sa multiplicité la création étrangère? De même pour le critique littéraire qui, partant d'une poétique faussée, risque de s'orienter sur des voies autres que celles ouvertes par l'original. D'où les réticences bien connues de la littérature comparée, par exemple, à œuvrer sur des corpus traduits. En ce qui concerne Faulkner, la preuve n'est plus à faire à propos du

17. Henri Meschonnic, Pour la poétique II (Paris, NRF/Gallimard, 1973), p. 420.

18. Ibid. p. 353. 
gommage du comique de son cuvre $^{19}$. Par contre, le problème demeure encore très actuel et n'a rien perdu de son acuîté en ce qui concerne les sociolectes. Dans le chapitre suivant nous examinerons l'incipit du Bruit et la fureur, et essaierons d'évaluer les conséquences du choix du traducteur face à un type particulier de sociolecte, le "vernaculaire noir américain»" ${ }^{20}$. Afin de limiter l'ampleur de notre travail, nous ne nous intéresserons qu'à un aspect limité de la mimésis verbale, dans un seul de ses volets, les dialogues. De ce fait, nous centrerons notre étude sur la production d'un traducteur, non des moindres, Maurice-Edgar Coindreau ${ }^{21}$. Son travail a été consacré, si

19. Voir à ce sujet l'article d'Annick Chapdelaine: «L'échec du Faulkner comique en France: un problème de réception», Meta, XXXIV, 2 , 1989, pp. 268-279.

20. Nous empruntons cette expression à William Labov, Sociolinguistique (Paris, Editions de Minuit, 1976), tout en demeurant parfaitement conscient qu'il n'existe pas qu'une seule forme de VNA. Pour les besoins de notre essai nous cataloguerons comme VNA le parler des Noirs du Sud.

21. À titre indicatif, nous donnons, ci-dessous, la liste des principales traductions effectuées par Coindreau, de l'américain au français. Coindreau traduisait aussi de l'espagnol.

Titres

1. Manhattan Transfer

2. L'adieu aux armes

3. Le soleil se lève aussi

4. Tandis que $\mathrm{j}$ 'agonise

5. Lumière d'août

6. Le petit appent du bon Dieu

7. La route au tabac

8. Le bruit et la fureur

9. Treize histoires

10. Des souris et des hommes

14. Les domaines hantés

22. La sagesse dans le sang

23. La proie des flammes
Auteurs

John Dos Passos 1928

Emest Hemingway 1932

E. Hemingway 1933

William Faulkner 1934

W. Faulkner 1935

Erskine Caldwell 1936

E. Caldwell 1937

W. Faulkner 1938

W. Faulkner 1939

En collab. avec R.-N. Raimbault

John Steinbeck 1939

Truman Capote 1949

Flannery O'Connor 1959

William Styron $\quad 1962$ 
l'on peut dire, par la publication du premier tome des auvres de Faulkner dans la Pléiade en $1977^{22}$. Ce sera notre texte d'appui.

2. Le vernaculaire noir américain: une sémiotique neutralisée. Étude des cas de Luster et de Dilsey dans le Bruit et la fureur.

\section{A. Luster}

Comme nous l'avons précisé, notre étude porte sur la mimésis verbale (prise dans le sens restreint de dialogue) ${ }^{23}$. De façon à procéder à la mise en place des enjeux qui nous préoccupent, passons à l'analyse linguistique du premier d'entre eux dans le Bruit et la fureur.

«Écoutez-moi ça, dit Luster. A-t-on idée de se conduire comme ça, à trente-trois ans! Quand je me suis donné la peine d'aller jusqu'à la ville pour vous acheter ce gâteau. Quand vous aurez fini de geindre. Vous n'pourriez pas m'aider à trouver ces

$\begin{array}{lll}\text { 24. Les larrons } & \text { W. Faulkner } & 1964 \\ \begin{array}{l}\text { 29. La belle vie } \\ \text { 30. Les confessions de }\end{array} & \text { Dos Passos } & 1968 \\ \begin{array}{l}\text { Nat Tumer } \\ \text { 31. L'arbre aux souhaits }\end{array} & \text { W. Styron } & 1969 \\ & \text { W. Faulkner } & 1969 .\end{array}$

Nous constatons aisément le poids de son travail: les noms les plus prestigieux de la littéranure américaine sont passés par sa plume.

22. Faulkner, CEuvres romanesques, Tome 1, Sartoris, le Bruit et la fureur, Appendice Compson, Sanctuaire, Tandis que j'agonise. Bibliothèque de la Pléiade. Texte traduits par M.-E. Coindreau, H. Delgove, R.-N. Raimbault, revus par M. Gresset. Édition présentée et annotée par M. Gresset (Paris, Gallimard, 1977).

23. Bakhtine ramène à trois catégories les procédés de création de l'image du langage dans le roman: 1) l'hybridisation; 2) l'interrelation dialogisée des langages; 3 ) les dialogues purs. C'est cette dernière catégorie uniquement que nous étudions. Op. cit., 1978, p. 175. 
vingt-cinq cents pour que je puisse aller voir les forains, ce soir? ${ }^{24}$ 》

C'est Luster qui s'exprime ici. Sans être l'un des protagonistes essentiels, il jouit d'un certain statut, ne serait-ce que par le nombre de répliques qui lui sont octroyées dans cette première partie du roman, le 7 avril 1928, ainsi que dans la dernière, le 8 avril 1928. Il est important de noter que lorsque Luster ouvre la bouche pour prononcer ces mots, aucun «discours d'escorte évaluatif»" ne l'a présenté au lecteur, qui ne connaît de lui que son nom et ne peut le juger, longtemps, que par ses propos. La compétence verbale du personnage aura donc pleine valeur d'informant textuel. Voyons ce que l'examen de celle-ci révèle puisque, si nous en croyons Barthes, «l'homme est offert, livré par son langage, trahi par une vérité formelle qui échappe à ses mensonges intéressés ou généreux». ${ }^{26}$

1. Phonétiquement, à part les marques inévitables de l'oralité, répétition de "ça», interrogation à valeur phatique, exclamation, l'énoncé est correct. Il ne comporte qu'une élision, celle du «n'pourriez», parfaitement acceptable dans la langue parlée mais qui, dans ce contexte autrement très articulé, surprend. Cette apostrophe ici fonctionnera avant tout comme indicateur d'oralité.

2. Syntaxiquement, d'évidence, nous remarquerons la correction des conjugaisons: conditionnel de pouvoir; «pour que» suivi du subjonctif; la tournure «Quand + verbe» marquant le reproche irrite, reprise deux fois, dont une avec un futur antérieur indiquant moins un rapport de temps que l'attente courroucée de Luster. Tous ces verbes sont à la deuxième personne du pluriel - et nous savons combien la

24. Le Bruit et la fureur, La Pléiade, Éditions Gallimard, 1977, p. 351. Traduction revue par le traducteur en collaboration avec M. Gresset.

25. Philippe Hamon, Texte et idéologie (Paris, PUF, 1984), p. 25.

26. Roland Barthes, le Degré zéro de l'écriture (Paris, Gallimard, 1953 et 1972), p. 59. Nous en profitons pour rappeler cette autre formule barthésienne: «Chaque homme est prisonnier de son langage: hors de sa classe, le premier mot le signale, le situe entièrement et l'affiche avec toute son histoire.» Ibid. 
maitrise de cette conjugaison pose de problèmes à ceux qui manient mal ou approximativement le français. Luster vouvoie donc fort correctement son interlocuteur, soit respect, soit distance, soit politesse à son égard. Or, abstraction faite de la raison de son utilisation, il est indéniable qu'un rapport de hiérarchie, autant que de courtoisie, s'établit dès l'instant où un locuteur choisit le vouvoiement.

3. Sémantiquement enfin, nous noterons le «geindre», terme qui, sans être excessivement recherché, marque néanmoins une certaine préciosité de langage. Au quotidien, plus prosaiquement, nous nous «plaignons», nous «pleurons», nous «râlons» ou même, nous «chialons». Nous ne geignons pas. Dans le même ordre d'idée, nous n'allons pas «voir les forains». Si, à la rigueur, nous allons à la «fête foraine», plus couramment, nous nous rendons «à la fête», «à la foire» ou, ce qui eût été plus approprié ici, «au cirque».

Terminé cette analyse, quel portrait pourrions-nous tracer de Luster? Nous savons par Philippe Hamon que le «savoir-dire» constitue l'une des quatre relations privilégiée de l'effet-idéologie attaché à tout personnage, son discours étant un signe de sa valeur. Aussi, les propos «articulés» du jeune Luster «signifient». Ils signifient un garçon poli (vouvoiement), posé (prononciation entière), instruit (choix des mots, conjugaisons), bref, un jeune homme de relativement «bonne famille». Toutes ces constatations seront confirmés par sa seconde prise de parole:

"Venez, dit Luster. Nous avons assez cherché ici. Ils ne vont pas revenir tout de suite. Descendons au ruisseau pour trouver cette pièce avant que les nègres mettent la main dessus.»

Or nos constatations, en parfaite conformité avec le texte d'arrivé, deviennent pour le moins incongrues si nous les mettons en parallèle avec le texte de départ.

"Listen at you, now." Luster said. "Aint you something, thirty-three years old, going on that way. After I done went all the way to town to buy you that cake. Hush up that moaning. Aint you going to help me find that quarter so I can go to the show tonight." 
"Come on" Luster said. "We done looked there. They aint no more coming right now. Lets go down to the branch and find that quarter before them niggers finds it."

L'image verbale du personnage differe totalement. Les indices (surtout syntaxiques: aint, after I done went, aint you going to help me, they aint no more, before them niggers finds it /un seul indice sémantique niggers), qui tranchent par rapport à l'usage normatif, énoncent un garçon d'un niveau d'éducation sensiblement inférieur à celui que nous avons rencontré en français. La traduction, qui établit ainsi une dissonance interlinguistique, se situe à la fois au-delà et en-deçà de l'identité du personnage faulknérien. Au-delà car sa voix française, plus achevée, décapée de ses aspérités, s'est embourgeoisée, peaufinée; en-deçà car la voix anglaise n'était pas qu'individuelle. C'était en même temps une voix collective : celle de la communauté noire qui, posée en anglais, disparaît en français.

Tandis que tout anglophone parcourant la version faulknérienne pouvait situer Luster (sans l'aide de la narration) dans son milieu socio-économique et géographique, le lecteur français pour sa part devra attendre, associer, recouper, deviner, avant de parvenir à un contour plus distinct de Luster, sans que jamais le signe ainsi obtenu ne soit pour autant un informant textuel égal à celui de son original. Car la voix collective ne se livrait pas dans le contenu, qui reste bien le même, mais dans la forme. N'oublions pas qu'un texte est le sens de ses formes autant que le sens de ses mots ${ }^{27}$. Retrancher cette forme, c'est retrancher du sens, comme nous venons de le constater.

Certains pourraient faire remarquer, non sans raison, que la négritude de Luster n'est pas, à priori, si évidente. En réalité, le lecteur américain ne peut s'y tromper. Même s'il est à prime abord désarçonné par l'appellation «nigger» que son histoire nationale lui aura appris à attendre d'un Blanc plutôt que d'un Noir, le préverbal «done» (I done went, we done looked), la double négation (aint no more) et l'objectif

27. Meschonnic, op. cit. p. 420. «Toute traduction qui privilégie l'idée (même si, plus subtilement, elle croit associer la pensée et l'expression, elle les a d'abord disjointes, et les termes mêmes le disent) ne peut que se cogner aux formes dans sa propre cécité.» 
remplaçant le nominatif (before them niggers finds it) orientent considérablement son décodage. Pour peu qu'il ait écouté des Noirs parler, qu'il ait l'habitude du Sud, de sa littérature, ou mieux encore de Faulkner, il reconnaîtra dans ces indices autant de formules identifiant les Noirs_plutôt que les pauvres Blancs, par exemple. S'il lui reste des hésitations, Luster se chargera rapidement de les lever par sa troisième prise de parole. Son Here they is ne laisse plus planer aucun doute.

"Shut up that moaning," Luster said. "I cant make them come if they aint coming, can I. If you dont hush up, mammy aint going to have no birthday for you. If you dont hush, you know what I going to do. I going to eat that cake all up. Eat them candles, too. Eat all them thirty-three candles. Come on, let's go down to the branch. I got to find my quarter. Maybe we can find one of they balls. Here. Here they is. Way over yonder. See." [...] "See them. They aint coming back here no more. Come on. ${ }^{28 n}$

En fait, les propos de Luster sont doublement révélateurs. Non seulement nous confirment-ils son origine, mais en plus ils divulguent certains des procédés techniques dont usera l'auteur pour représenter les Noirs. Procédés encore bien circonspects à ce stade : nous n'en sommes qu'à la deuxième page et l'écrivain, conscient des contraintes de la lisibilité (qu'il a déjà fortement malmenée), évite la surcharge dialectale $^{29}$. Néanmoins, nous pouvons dès à présent formuler la remarque suivante : n'y figure pas un mot qui n'appartienne pas à

28. "Quand vous aurez fini de geindre, dit Luster. J'peux pas les faire revenir de force, hein? Si vous ne vous taisez pas, mammy n'fêtera pas votre anniversaire. Si vous ne vous taisez pas, savez-vous ce que je ferai? J'mangerai tout le gâteau. J'mangerai les bougies aussi. J'mangerai les trente-trois bougies. Venez, descendons au ruisseau. Faut que je trouve mon argent. Peut-être que nous trouverons une de leurs balles. Tenez, regardez, les voilàl Là-bas, au loin.» [...] «Vous voyez. Ils n'reviennent plus par ici. Venez.» Pléiade, p. 352.

29. Il suffit pour s'en convaincre de comparer avec les propos, autrement plus agrammaticaux, des mêmes personnages dans la dernière partie de The Sound and the Fury. 
l'anglais standard; aucun archaïsme, aucun argot. C'est uniquement dans la structure syntaxique agrammaticale que les propos de Luster établissent leur appartenance, leur «couleur» ${ }^{30}$. Ce que confirmeront à profusion les dialogues subséquents puisque, pour répondre aux contraintes de la lisibilité et de la cohérence, le texte enfermera très vite ces effets mimétiques dans la récurrence de marqueurs identiques, procédé dont l'aboutissement frise dangereusement la stéréotypification.

En effet, l'agrammaticalité typique du VNA constitue aussi l'indice d'un manque d'instruction, qui risque d'entraîner une accusation de prévention à l'encontre de l'écrivain auquel on reprochera une peinture stéréotypée et peu flatteuse des Noirs. Accusation portée par ceux qui ne verront dans ce parler que sa déviance, oubliant le passé et l'Histoire dont il est le témoignage. Ce serait en outre prêter un peu vite des intentions à un écrivain là où elles sont le plus difficilement attribuables. Car c'est dans ces moments de mimésis intense, où la fiction imite au plus près le réel, que l'auteur disparaît, irrepérable derrière (dans?) les propos du personnage. Lorsque Faulkner copie le langage des Noirs, sait-on jamais avec certitude «à partir de quel lieu cette énonciation s'opère: un lieu critique? Distant? Empoissé? » ${ }^{31}$

Néanmoins, si l'auteur s'estompe, le texte demeure, et nous pouvons opposer les valeurs diégétique et sémiotique à la valeur grammaticale. L'utilité du bavardage de Luster ne réside pas uniquement dans ce qu'il nous révèle sa race : il est, de plus, indispensable au roman. Sans lui, le lecteur, égaré dans les fragments épars de réalité dispensés par une conscience atrophiée (Benjy), ne pourrait rassembler les pièces du puzzle. Luster, par son soliloque, axe notre compréhension. Le cirque est en ville; c'est l'anniversaire de

30. Les études linguistiques sur le vemaculaire noir insistent elles aussi sur l'importance de la syntaxe dans sa construction. On se reportera en particulier aux travaux de J.L. Dillard, Black English, Its History and Usage in the United States: "Syntax, the focus of more modem linguistics, is the area in which the analysis of Black English is most revealing." (New York, Vintage Books, 1973), p. 40.

31. Barthes, le Bruissement de la langue, Essais critiques IV (Paris, Seuil, 1984), p. 116. 
Benjy; il a trente-trois ans; il est retardé, d'où le manque de suite des scènes. Il s'ensuit que si le récit est généré par l'esprit chaotique du jeune Blanc, c'est par le verbe du jeune Noir qu'il acquiert une certaine cohérence. Évidemment, la traduction respectueuse du fond ne transige pas sur le sens littéral et tous les jalons sont reproduits. Cependant, ce ne sera plus une voix noire qui orientera le lecteur.

\section{B. Dilsey}

Dilsey est sans nul doute le principal «actant» noir du roman ${ }^{32}$. Pourtant, ses «actes» n'outrepassent jamais ses fonctions: elle agit très peu et ne motive aucun des axes diégétiques, rôles dévolus à Caddy, Jason et Quentin. Toutefois, si à proprement parler Dilsey n'agit pas, elle «réagit», par son verbe plus que par son faire, aux sollicitations environnantes immédiates. Elle ne «pense» pas non plus puisque le récit, qui pourtant ne se prive pas d'accorder des courants de pensée, ne lui concède aucune forme de réflexion. Ce qui coïnciderait avec les tendances du roman sudiste américain.

[Only a handful of white Southern writers] have attempted stream-of-consciousness techniques, deep psychological probing, or an exclusively Negro point-of-view. Notice that it is a standard critical commentary on The Sound and the Fury that Faulkner fails to give Dilsey a stream-of-consciousness section parallel with that of each of the other main characters. Apparently he felt incapable of putting himself in the place of the Negro, although he chanced identifying with a woman and

32. Coindreau le reconnaît d'ailleurs lui-même puisque prenant la défense de Faulkner contre les détracteurs de The Sound and The Fury, il écrivait: "But the censors forgot that the most important character in the book is Dilsey, undoubtedly one of the most exemplary figures of a woman ever to appear in fiction." George McMillan Reeves, éd. The Time of William Faulkner. Essays by Maurice Edgar Coindreau, with a Foreword by Michel Gresset. (Columbia, University of South Carolina Press, 1971), p. 20. 
an idiot - a striking commentary on his belief in the remoteness of Negro psychology. ${ }^{33}$

Sa place actantielle aurait pu être minime si le narrateur n'avait accordé tant d'importance à l'espace discursif, la valeur actantielle tenant ici autant dans le dire que dans le faire, sinon plus. Et dans cet univers du verbal, heureusement pour elle, Dilsey parle, et beaucoup même. Son champ discursif est autrement plus vaste que celui de Mrs Compson ou de son mari; elle est la seule, avec Jason, à avoir un contact oral continu du début à la fin du roman avec tous les autres membres de la maisonnée, blancs ou noirs. Elle figure dans la plupart des scènes importantes, que d'ailleurs elle ne se prive pas de commenter, avec sa verve, son humour et sa franchise habituels, car Dilsey dit ce qu'elle pense. Il suffit d'une brève incursion dans les dialogues pour nous en convaincre. Écoutons-la, tour à tour, donner la réplique aux principaux acteurs. Tout d'abord avec son mari Roskus qui se plaint de sa condition et de la présence de Benjy:

"It aint hurt none of you and yourn, is it. Versh working and Frony married off you hands and T.P. getting big enough to take your place when rheumatism finish getting you." (p. 34)

Avec Mrs Compson qui lui dit de se hâter:

"I'm coming just as fast as I can" Dilsey said. "She ought to know by this time I aint got no wings." (p. 36)

Avec Luster qui veut saisir une autre tranche de gâteau:

"Reach it again, and I chop [your hand] right off with this here butcher knife." Dilsey said. "I bet he aint had one piece of it." [...] "Reach hit one more time." Dilsey said. "Just reach it." (p. 70)

Avec Caddy à propos du changement de nom de Benjy:

33. Nancy M. Tischler, Black Masks, Negro Characters in Modern Southern Fiction. (The Pennsylvania State University Press, 1969), p. 16. 
"Huh, Dilsey said. Names aint going to help him. Hurt him, neither. Folks dont have no luck, changing names. My name been Dilsey since for I could remember and it be Dilsey when they's long forgot me." (p. 71)

À Jason qui refuse le droit de visite à Caddy:

"You's a cold man, Jason, if man you is," she says. "I thank de Lawd I got mo heart dan dat, even ef hit is black." (p. 258)

Ou lorsque ce dernier lui lance "Did you hear me?", la réponse ne se fait pas attendre:

"I hears you," Dilsey said. "All I been hearin, when you in de house. Ef hit aint Quentin er yo maw, hit's Luster en Benjy. Whut you let him go on dat way fer, Miss Cahline?" (p. 346)

À Mrs Compson qui lui dit de faire moins de bruit pour ne pas réveiller Jason:

"I dont see how you expect anybody to sleep, wid you standin in de hall, holl'in at folks fum de crack of dawn..." (p. 337)

Et lorsqu'elle lui propose de descendre l'aider à la cuisine pour préparer le petit-déjeuner, Dilsey rétorque:

"En who gwine eat yo messin?" Dilsey said. "Tell me dat. Go on now", she said. (p. 338)

La liste pourrait continuer. Néanmoins, nous constatons vite que si Dilsey n'est pas la meilleure grammairienne au monde, tant s'en faut, elle n'en incarne pas moins la voix de l'authenticité et du bon sens; celle qui donne à chaque personnage le contrepoint verbal critique de ses actes, avec une verdeur non dénuée de drôlerie ni d'astuce. Ainsi, pour la servante noire spoliée de toute initiative sociale et textuelle, il ne reste pour se défendre ou, plus fondamentalement, pour exister, que son verbe. C'est par lui qu'elle accède à l'existentiel, et nous ne pensons pas, dans ces conditions, que l'éviction de sa couleur soit un 
détail ${ }_{i}$ de «moindre importance» ${ }^{34}$. En outre, si en elle s'incarne la voix de la sincérité, comment la sémiotique œuvrant en français retrouvera-t-elle la dichotomie initiale séparant le dire du faire; celle où la valeur morale du personnage se livre selon une modalité inverse à sa correction linguistique ${ }^{35}$ ? où l'authenticité et l'abnégation se lisent à travers les aphérèses, apocopes ou syncopes des structures langagières?

Le gommage des aspérités langagières de Luster et de Dilsey constitue une éviction autrement plus grave que celle d'une simple teinte exotique. Les Noirs sont irrémédiablement circonscrits dans une correction langagière neutralisant toute variante et originalite $e^{36}$. Appliquée uniformément, cette éviction entraîne la disparition totale de

34. Voir à ce propos à la page 174 (note 41) la citation de Coindreau.

35. Nous retrouvons une tendance un peu analogue dans Manhattan Transfer où ceux qui manient le mieux l'anglais se situent de façon générale dans l'isotopie des valeurs dégradées (ambition, mercantilisme, cupidité, snobisme, oisiveté, duplicité, mensonge, fourberie, etc.), alors que les immigrants ou les pauvres bougres qui s'expriment dans un anglais patoisant ou peu correct, peuplent l'isotopie des valeurs authentiques (travail, amitié, honneur, fraternité, solidarité, etc.).

36. Aucune modification de tonalité n'est prise en compte dans la traduction. Pourtant, le TD nous offrait des exemples d'un changement dans la parole d'un même personnage, comme dans le cas du Deacon (Vintage p. 120-124; Pléiade p. 433-435), et celui du révérend Shegog (Vintage p. 365-370; Pléiade p. 607-610). De plus, c'est faire bien peu de cas du hors-texte historique. L'apprentissage de la langue chez les esclaves ou les indigènes s'est accompli avant tout oralement par mimétisme ou «psittacisme», ce que la représentation anglaise confirme. En aucun cas par l'étude formelle, ce que le langage corrigé de Dilsey aurait exigé. Le Noir ne pouvait que reproduire ce qu'il entendait or, chaque fois que son maître lui parlait, il devenait un «tu»». Aussi, dans les pays qui furent à une époque colonies françaises le tutoiement est-il fréquent de la part des habitants; le vouvoiement y est plus rare, et le soin de personnes instruites maîtrisant parfaitement la conjugaison des verbes français. Ce que laisse supposer la Dilsey française. 
tout le réseau des voix noires, et élimine de la sorte, purement et simplement, l'altérité du groupe. Son identité. Sa négritude.

Pourtant, le texte de départ, ne se privait pas d'étaler cette altérité, d'autant plus sensible qu'elle s'appréhendait aussi dans son opposition au parler des Blancs, la valeur du signe étant différentielle. D'où la saveur des propos des Noirs sàisis, par contraste, selon les arêtes les plus vives de leur altérité, que ce contraste soit issu d'un voisinage immédiat, ou d'une scène analogue reprise en contrepoint. Les exemples du premier cas sont foison. Ainsi de l'échange entre Caddy et Frony :

"Where do you and T.P. have the measles, Frony."

"Has them just wherever we is, I reckon.» Frony said. (Vintage:45)

Ou entre T.P., Caddy et Frony:

"I skeered I going to holler." T.P. said. "Git on the box and see is they started.»

"They haven't started because the band hasn't come yet." Caddy said.

"They aint going to have no band." Frony said.

"How do you know." Caddy said.

"I knows what I knows." Frony said. (Vintage:45).

Entre Dilsey et Mrs Compson:

"All right," Dilsey said, "All right, here I is. I'll fill hit soon ez I git some hot water." [...] "Put hit down dar en g'awn back to bed."

"I couldn't understand what was the matter," Mrs Compson said. "I've been lying awake for an hour at least, without hearing a sound from the kitchen."

"You put it down and g'awn back to bed," [...] "I'll have de fire gwine in a minute, en de water hot in two mo."

"I've been lying there for an hour, at least," Mrs Compson said. "I thought maybe you were waiting for me to come down and start the fire." (Vintage:333). 
Le roman entier est jalonné de ce genre de confrontations dont le relevé complet serait trop long, et fastidieux.

Pour le deuxième cas, nous citons un type d'échange dont la récurrence dans la première et la dernière partie finit par lui octroyer la valeur d'un tableau: Benjy hurle et son gardien (Caddy/T.P./Luster) se fait réprimander par un adulte (la mère/Dilsey/Jason).

"What is the matter now." Mother said. "Benjamin."

"Benjy" Caddy said. [...]

"You must have done something to him." Mother said. "Why wont you let him alone, so I can have some peace. Give him the box and please go on and let him alone." (Vintage:49).

"What you done to him now." She [Dilsey] said.

"Aint done nothing to him." Luster said. "He just started bellering."

"Yes you is" Dilsey said. "You done something to him. Where you been."

"Over yonder under them cedars." Luster said. (Vintage:67).

Cette valeur différentielle des langages permet en outre au lecteur anglophone de saisir les alternances, les mouvances, les changements de ton, les télescopages - ou, au contraire, l'absence de ceux-ci. Car, ces langages qui se frôlent sans cesse, quasiment à chaque page, signalent leur attitude respective dans leur énonciation. Nous constatons alors que s'il y a échange de contenu informationnel, il n'y a pas nécessairement échange de forme. Les langages, observés et rapportés de façon monologique, se côtoient, coexistent mais ne s'interpénètrent pas - pas même ironiquement. De l'un à l'autre, c'est l'incommunicabilité. Miss Caroline parlant à Dilsey, le père s'adressant à Versh, Quentin au Deacon, rêfractent dans l'étanchéité respective de leur langue celle qu'ils vivent quotidiennement dans leur rapport social. Le langage, qui n'est jamais pris dans une dialectique, devient dès lors une sorte de mise en abyme du social où la valeur sémiotique des espaces discursifs reprend celle que l'on constatait, par exemple, dans la répartition des lieux diégétiques. «L'image révèle ici non seulement 
la réalité, mais les virtualités du langage donné, ses limites idéales, si l'on peut dire, son sens total, cohérent, sa vérité et sa limitation» ${ }^{37}$.

Il est intéressant de noter à ce propos que seuls les enfants Compson migrent librement de l'espace réservé aux Noirs à celui des Blancs. De même, seule la langue des enfants acceptera des tonalités noires, aussi rares soient-elles. Nous verrons Caddy (Vintage:28) reprendre une expression entière de Dilsey, avec sa prononciation particulière de «Lord», «Lawd»; Jason aussi acceptera l'influence de ses compagnons de jeux, surtout dans ses moments de colère et de frustration. Le parler de leurs parents quant à lui demeurera hermétique à celui des domestiques. Il s'en démarquera même nettement par le soin qu'ils apportent à leur expression, quelle que soit la circonstance.

Mother began to cry. "If you begrudge Maury your food, why aren't you man enough to say so to his face. To ridicule him before the children, behind his back"

"Of course I dont." Father said. "I admire Maury. He is invaluable to my own sense of racial superiority. I wouldn't swap Maury for a matched team. And do you know why, Quentin."

[...]

"Et ego in arcadia I have forgotten the latin for hay." Father said.

[...]

"Of course." Father said. "Bad health is the primary reason for all life. Created by disease, within putrefaction, into decay. Versh." (Vintage:52-53).

Orné d'une citation latine, d'un rythme sentencieux, d'un vocabulaire plus abstrait, le verbe paternel tranche fortement sur celui du locuteur qui suit à la même page, quatre lignes plus bas, nulle autre que Dilsey.

"You a big boy." Dilsey said, "Caddy tired sleeping with you. Hush now, so you can go to sleep."

37. Bakhtine, op. cit., 1978, p. 173. 


\section{[...]}

"Ain't you going to be a good boy and hush." Dilsey said.

"You aint, is you. See can you wait a minute, then."

La différence est on ne peut plus nette.

Le rapport social, qui est aussi un rapport au pouvoir, passe par le verbe. L'écrivain, dont la conscience plurilingue n'est plus à mettre en doute, concrétise dans le panorama sociolectal qu'il nous donne à lire, l'enjeu des rapports sociaux. Dans The Sound and the Fury, les langages, qui «tendent vers la limite de l'incompréhension mutuelle des individus parlant des langues différentes», ${ }^{38}$ peuvent se croiser ou se cotoyer mais, pas plus que les êtres, ils ne sauraient se métisser.

\section{Coindreau, l'institution littéraire française et le vernaculaire noir:} la problématique de l'éviction.

Pour que le refus du métissage soit perceptible, il aura fallu que préalablement les deux entités langagières aient été mises en contact; le signe résultant de leur disposition l'une envers l'autre. Or, en français, une telle possibilité ne s'envisage même pas puisque, comme nous l'avons vu pour Luster et Dilsey, les entités sont éliminées d'emblée, chaque langage se fondant dans un même moule normatif, bourgeois, «classique» ${ }^{39}$. Et même si, avec l'aide du récit, le lecteur parvient à déterminer l'ethnicité des différentes voix, pourra-t-il être absolument certain de ses répartitions? Dans les premières pages, où placera-t-il Versh, T.P. et Dilsey que rien ne distingue dans leur verbe francisé, alors qu'en anglais ils sont incontestablement Noirs? Pourquoi la traduction refuse-t-elle toute représentation graphique de leur origine?

38. Bakhtine, op. cit., 1978, p. 173.

39. Le terme «bourgeois» semble peut-être excessif: nous verrons qu'il n'en est rien. On se reportera sur ce sujet à l'analyse qu'A. Chapdelaine trace de la réception de Faulkner en France dans l'entredeux-guerres (loc. cit., 1989), en particulier dans le rapport époquesociété-classe (p. 273). 
S'agit-il d'une impossibilité inhérente à la langue ${ }^{40}$ ? d'un constat inconscient d'intraduisibilité?

Écoutons le traducteur, M.-É. Coindreau, s'expliquer sur les raisons de son choix.

I have often been asked, "How can you translate dialect?" This is, in my opinion, a detail of slight importance. If the country people in Faulkner's work speak a Mississippi dialect, they speak above all as country people do, and nothing else matters. The same reasoning may be applied to Negroes. If Dilsey, the admirable «mammy» of the Compson family in The Sound and the Fury, retains our attention, it is not because of the color of her skin. What makes her a great figure of fiction is the nobility of her character, her qualities of devotion, abnegation, and endurance, all of them qualities which can be rendered in any language without detracting in the least from Dilsey's greatness. All men of my generation have known in the homes of their parents and their grandparents white counterparts of Dilsey. We know how they spoke and this is the thing that concerns $u^{41}$.

Comme il y avait lieu de s'y attendre, son parti-pris résulte donc moins de la reconnaissance d'une impossibilité qui, à la rigueur, eût pu se comprendre puisque, nous dit Meschonnic, «pour une Euvre donnée dans un rapport interlinguistique-interculturel donné, l'interaction des poétiques et la ré-énonciation historique peuvent ne s'être pas encore produites, peuvent ne pas se produire», ${ }^{42}$, que d'un acte délibéré d'indifférence posant l'altérité de l'Autre comme un détail de peu d'importance. Ce qui nous semble plus grave. N'en déplaise à $\mathrm{M}$. Coindreau, Dilsey n'est pas une servante vend́enne, et elle retient notre attention non seulement parce qu'elle est la servante universelle, mais parce qu'elle est descendante d'esclaves, femme, servante, et Noire. La

40. La question n'est évidemment que rhétorique.

41. The Time of William Faulkner, p. 89. C'est nous qui soulignons.

42. Meschonnic, op. cit., p. 309. 
problématique de son existence ne saurait se confondre avec celle d'un modèle censément universel, en réalité étroitement régional, et justifiable dans le redoublement d'un vécu; modèle inspiré par une conception subjective et annexionniste de la traduction, selon lequel il ne s'agirait plus que de susciter une évocation par contiguité, en annexant l'étranger au même, au déjà-entendu, le nouveau à l'ancien. Sans compter que le traducteur valorise abusivement l'illusion du naturel, «comme si [le] texte en langue de départ [avait été] écrit en langue d'arrivée, abstraction faite des différences de culture, d'époque, de structure linguistiquen ${ }^{43}$.

N'oublions pas toutefois que les positions théoriques et les pratiques sont historiquement situées ${ }^{44}$. De sorte que la synchronie du traducteur (le Bruit et la fureur a été publié par les éditions Gallimard en 1938), son rang social, sa profession - diplômé universitaire, professeur de lettres françaises à Princeton, en filiation directe aux courants du siècle précédent et imbu d'une culture classique qui le rendait sourd aux langages sociaux - , permettent de comprendre ses censures $^{45}$. Adepte du «bon français», selon M. Michel Gresset, «Coindreau se refuse à l'évidence à écrire quoi que ce soit qui ne satisfasse à deux critères d'ailleurs souvent confondus, celui du «bon» français (notion évidemment normative) et celui d'un certain cartésianisme ('Ce qui se conçoit bien $s^{\prime}$ énonce clairement') ${ }^{46}$. La

43. Ibid., p. 308.

44. Meschonnic, op. cit., p. 322.

45. D'ailleurs M. Coindreau est un homme qui n'hésitait pas à utiliser le passé simple dans sa conversation. Il suffit de relire ses entretiens avec Christian Giudicelli (diffusés sur France-Culture en 1971) pour en avoir la preuve. Ces occurences de la surnorme, même dans une émission culturelle, ne perdent rien de leur incongrüté dans la langue parlée. Voir Maurice-Edgar Coindreau, Mémoires d' un traducteur, Entretiens avec Christian Giudicelli (Paris, NRF/Gallimard, 1974).

46. M. Gresset. Aspects de la traduction littéraire: Poe et Baudelaire, Faulkner et ses interprètes, SSLM-Annuario, n.1 (Trieste, 1985), p. 72. 
conséquence étant que la traduction sera souvent plus claire que l'original, et aussi plus réfractaire aux déviances, sociolectales ou autres.

Une telle attitude, nécessairement historique, ne ressortirait plus seulement à l'individuel, comme nous l'avons déjà remarqué : il s'agirait d'un cas de société, et il nous serait facile de déceler ces «critères» dans la majorité de la production littéraire française, dont le classicisme n'est plus à prouver. Cette perspective trouvera d'ailleurs involontairement son écho dans une constatation de Barthes:

Le roman, dès lors qu'il est devenu réaliste, a fatalement rencontré sur son chemin la copie des langages collectifs; mais en général l'imitation des langages de groupe (des langages socio-professionnels) a été déléguée par nos romanciers à des personnages secondaires, à des comparses, chargés de «fixer» le réalisme social, cependant que le héros continue de parler un langage intemporel, dont la «transparence» et la neutralité sont censées s'accorder à l'universalité psychologique de l'âme humaine ${ }^{47}$.

Les traductions de Coindreau ne feraient donc que réfléchir les tendances de la littérature de son époque:

La notion de traduction est une notion historique. La traduction comme pratique idéologique courante (non théorisée, philologisée, esthétisée), qu'on a appelé traduction-introduction, ou traduction-traduction ou traduction non-texte, par opposé à la traduction-texte se définit par le possible d'une époque. Le possible d'une époque est la somme de ses idées reçues ${ }^{48}$.

Ce qui est une forme comme une autre d'exonération du parti pris adopté par Coindreau. Pourtant, l'affaire ne saurait être si simple. Il se trouve que la synchronie en question avait fourni la preuve, un an auparavant, qu'il ne s'agissait nullement d'une impossibilité linguistique

47. Barthes, op. cit., 1984, pp. 114-115. C'est nous qui soulignons.

48. Meschonnic, op. cit., p. 321. 
ni même sociale, et que l'on pouvait bel et bien «recréer» une évocation du vernaculaire noir américain en traduction. En effet, un autre texte de Faulkner, Sartoris, avait paru aux mêmes éditions Gallimard en 1937, et ses traducteurs, R.-N. Raimbault et H. Delgove, ayant opté pour un point de vue différent, avaient fait la démonstration de cette possibilité: leur texte autorisait par des séries de marqueurs précis la perception de la «négritude» des personnages ${ }^{49}$. Certes, leur évocation pouvait être jugée plus ou moins satisfaisante, plus ou moins réussie : il n'empêche que la pratique adoptée par Raimbault-Delgove laissait au lecteur, et lui seul, le soin de juger de l'effet produit.

Hélas, il semblerait que l'institution littéraire en eût jugé autrement, préférant emboiter le pas à Coindreau plutôt qu'aux «vernaculairisants», et dire sans équivoque au lecteur ce qu'il lui fallait penser d'une telle représentation. Coindreau évidemment fut le premier: nous avons déjà pu lire ses commentaires, publiés pour la première fois en 1960. Il s'est trouvé par la suite un allié puissant en Michel Gresset, lui-même professeur d'université, traducteur, et faulknérien de renom. Celui-ci, dans son avant-propos à l'ouvrage consacré aux essais critiques de Coindreau, a non seulement endossé le point de vue de son aîné mais, en plus, s'est attaqué à la position adverse de Raimbault-Delgove.

He [Coindreau] has always held the view that Dilsey in The Sound and the Fury, was not so much a Negro woman as the perennial house servant, the kind he had been extremely familiar with since his childhood in Vendée. He, therefore, had no trouble translating her speech, for unlike less tactful translators of Faulkner, he avoided the pidgin French of the West Indies for the good old speech of all the Dilseys of the world, whatever their color. But had he met with any trouble, he might only have reread Flaubert's Un coeur simple ${ }^{50}$.

49. Cette version, qui est encore accessible dans la version Folio de Sartoris, a été remaniée pour son insertion dans le tome de La Pléiade, comme nous le verrons plus loin.

50. The Time of William Faulkner. Avant-propos, xi. C'est nous qui soulignons. 
En quoi ces traducteurs sont-ils less tactful? La réponse n'est pas fournie. M. Gresset voyait-il dans ce pidgin French une forme de racisme? Craignait-il l'accusation d'une peinture défavorable? Dans le contexte social et historique français, il n'est pas interdit de penser que telle fut l'origine de sa retenue face au vernaculaire. On constatera en outre que Dilsey perd sa négritude alors même que Flaubert vient à la rescousse. N'y aurait-il pas là, en sus, l'indice d'une vision quelque peu annexionniste du traduire? Vision entérinant l'effacement de toute distance culturelle par la promotion de l'identification, et refusant de voir en Dilsey autre chose qu'une servante blanche? Quoi qu'il en soit, un texte plus récent du même auteur (1985), renchérit sur cette première prise de position, allant même encore plus loin:

Le choix décisif de Coindreau concernant l'usage du «tu» et du "vous» au sein de la famille Compson me parait mériter d'être considéré comme intouchable, même si le vouvoiement a presque disparu en France dans un tel contexte. En faisant vouvoyer les Blancs par les Noirs et les parents par les enfants, Coindreau a subtilement transposé des habitudes probablement ancrées dans le milieu bourgeois de sa Vendée natale; ce faisant, il a parfaitement compensé la perte, aussi énorme qu'inévitable, dans les dialogues des marques dialectales du parler noir. Le choix opposé de René-Noël Raimbault dans Sartoris apparaît, par contraste, insoutenable; notamment l'élision systématique du «r», pour tenter de figurer l'accent africain ou antillais, est devenu illisible - et ridicule: on évoque les publicités de Banania ${ }^{51}$.

Si les propos ci-dessus permettent de situer historiquement le parti pris adopté par Coindreau (nous n'avions pas tort de parler d'embourgeoisement en ce qui concerne Dilsey, Luster, T.P. et les autres), ils ont par contre, dans l'optique de la traduction, de quoi choquer. M. Gresset, dont le travail critique des ceuvres de Faulkner dans la Pléiade est admirable, assume ici une position qui nous paraît être bien spécieuse. Comment le vouvoiement, marque d'une maîtrise suffisante de la langue et indice, entre intimes, d'une civilité toute

51. M. Gresset, loc. cit., Trieste, 1985, pp. 77-78. C'est nous qui soulignons. 
bourgeoise, et désuète déjà en 1938, peut-il compenser le dialectal ou le vernaculaire noir? Comment la problématique de l'esclavage peut-elle se retrouver dans ce «vous» vendéen affecté? Il s'agit là, selon nous, d'un contresens de ton grave, d'une dissonance sociale, en même temps que d'un refus du changement puisque nous savons, par la plume même de l'intéressé, qu'il a pris une part active, en compagnie du traducteur, à la révision du texte du Bruit et la fureur dans le but de le «dépoussiérer». Pourquoi alors ne pas avoir saisi l'occasion d'infléchir la représentation verbale du côté de la négritude? Coindreau s'y opposait-il si farouchement?

Nous acceptons le fait qu'il était certainement difficile d'aller à l'encontre de la volonté du traducteur en ce qui concerne le Bruit et la fureur. Mais était-il par contre nécessaire de remanier systématiquement Sartoris dans un sens analogue? Car si la correction se contentait de rectifier le texte de Coindreau de facon somme toute assez superficielle ${ }^{52}$, elle n'hésitait pas par contre à reprendre celui de Raimbault-Delgove de façon plus systématique, allant même jusqu'à effaçer dans les dialogues toutes les marques sociolectales du VNA qu'elle jugeait inappropriées, édulcorant de la sorte considérablement leur version. La prestigieuse Pléiade exigeait-elle un texte «officiel» plus digestible, plus conforme aux normes? Redoutait-on là encore les accusations malveillantes? Quelles qu'aient pu être les raisons des éditeurs et de M. Gresset, l'essentiel est que le travail de ce dernier ainsi que son discours critique ultérieur, ont affirmé puis validé un état de fait qui aurait dû demeurer contestable - et être contesté. "The unacknowledged norms underlying much of the thinking about what "good" translations ought to be like, turn out to be awkwardly and arrogantly absolute formulations of something that is, in essence,

52. Les changements apportés, en vue de soi-disant actualiser la traduction, ne portent que sur des questions de détails sémantiques très ponctuels (le mot drugstore par exemple) qui ne changent en rien la poétique générale. Voir son article cité, Trieste, 1985. 
relative and transient: a poetics, the principles regulating the literary production of an era. $)^{53}$

Refusant, même dans une synchronie plus contemporaine, de prendre en compte la «réalité» noire, l'instance réceptrice-régulatrice a donc repoussé les «choix opposés» - oublieuse en cela du fait qu'une position historique change l'intraduisible - et s'est montrée particulièrement sévère, au nom du bon goût, de la lisibilité, et du ridicule, envers les traducteurs qui «ont osé» une figuration de l'accent noir. Or, cette illisibilité qui leur est reprochée est à tout le moins fort discutable, comme le prouve l'extrait ci-dessous de Sartoris. C'est Simon, le serviteur noir qui parle:

«Missié Baya'd l'est lentlé, débuta Simon sur un ton de conversation.[...] L'est allivé p' l'tlain d'deux heu'es, poursuivit-il. L'a sauté du côté où fallait pas et l'a filé pa' les bois. Y a un homme d'équipe qui l'a vu. S'ment, l'était point enco' allivé à la maison quand j'suis pa'ti. J'c'oyais des fois qu'il 'tait avec vous.»

53. André Lefevere, Literary Theory and Translated Literature. Dispositio (The Art and Science of Translation), Vol. III, $\mathrm{n}^{\circ}$ 19-20-21, 1982, pp. 3-22. Nous rejoignons ici une prise de conscience intéressante mentionnée par Lefevere dans son article, puis plus récemment par A. Brisset (op. cit., 1990). Il semblerait qu'il fût plus aisé de reconstituer les normes poétiques d'une époque en fonction des critères stylistiques imposés aux traductions qu'elle a produites, que sur la base d'une simple analyse de ses seuls textes (Lefevere, 1982, p. 7). La traduction provoque un «bougé» (Brisset, 1990, p. 29) qui, dans une certaine mesure peut être le résultat des règles poétiques du polysystème d'arrivée: «C'est en effet la régularité de certains choix qui permet d'identifier les éléments constitutifs de l'institution littéraire propre au milieu-cible, en particulier dans sa composante discursive. Les normes qui régissent les stratégies de traduction interviennent aussi bien avant que pendant le transfert. Elles agissent à des degrés divers, mais elles sont en grande partie déterminées par la fonction que les textes qu'on traduit doivent remplir dans le polysystème de la littérature d'arrivée, c'est-à-dire par la place que l'institution leur $y$ assigne.» 
On comparera ces quelques lignes avec la prise de parole de Simon dans l'original.

"Mist' Bayard done got home," Simon remarked in a conversational tone. [...] "He got offen de two o'clock train," he continued. "Jumped off de wrong side and lit out th'ough de woods. Section han' seed 'im. Only he ain't never come out home yit when I lef'. I thought he wuz wid you, maybe."

On est en droit de se demander lequel des deux textes est le plus illisible, ou ridicule ${ }^{54}$ ? La version de La Pléiade donne:

«Mr Bayard est de retour», laissa tomber Simon sur un ton dégagé. [...] «L'est arrivé par le train de deux heures, poursuivit-il. L'a sauté du côté ou il fallait pas et l'a filé par les bois. Y a un homme d'équipe qui l'a vu. Seulement il était pas encore arrivé à la maison quand j'suis parti. J'croyais des fois qu'il était p'têt avec vous.»

Nous ne cherchons pas à incriminer trop durement $M$. Gresset dont, nous l'avons déà dit, nous admirons l'appareil critique de ce tome 1. Cependant, en poussant plus loin notre analyse, nous ne pouvions que constater combien sa position devenait pour le moins surprenante, pour ne pas dire injustifiée. En effet, alors qu'il reprochait durement à R.-N. Raimbault et H. Delgove leur évocation «antillaise» par l'élision du $\langle(»$, certes un poncif graphique vomitoire à propos des Noirs, il fermait les yeux d'une part sur le fait que ces traducteurs ne s'en

54. Sartoris, édition Folio, pp. 15-16; La Pléiade, p. 6; Signet Classic, p. 22. «Le traduit aide à traduire ce qui n'est pas encore traduit. Certaines oeuvres, pour des raisons historiques objectives, n'étaient pas ou ne sont pas traduites encore. Ainsi Bakhtine montre que jusqu'à tel changement idéologique Rabelais passait pour intraduisible en russe. Lien entre les études sur un texte, leur substrat culturel, et les possibilités de lire-écrire ce texte. Puis Rabelais s'est mis à parler russe. Le traduire auparavant n'avait pas été possible, historiquement - et non par principe: mais l'intraduisible, hors de son histoire et av ant la venue de ces conditions culturelles de la traduction, passe par définition pour un principe.» Meschonnic, op. cit. p. 359. 
tenaient pas à cet unique procédé, et que leur représentation était plus complexe; d'autre part sur celui que l'un des principaux adeptes de l'élision n'était autre que Coindreau lui-même, puisque les rares moments où sa mimésis s'éveille véritablement aux tonalités noires sont ceux où disparaît le «r», précisément ${ }^{55}$. M. Gresset, respectueux du travail de Coindreau, a, semble-t-il, choisi de ne pas s'en offusquer. Pourtant, ici plus qu'ailleurs, ses reproches n'auraient-ils pas dô s'élever puisque par ce moyen, le seul, Coindreau cantonnait abusivement ses ressources aux choix de la place publique. Cette réintégration ultime, mimétisme compensatoire de surface, contredit (sans pour autant la neutraliser) la position initiale du célèbre traducteur de Faulkner, donnant à penser qu'en dépit de ses affirmations, celui-ci a senti que le gommage absolu ne pouvait s'envisager impunément; que «son langage unique résonnait néanmoins au milieu du plurilinguisme, et qu'il n'était pas incontestable ${ }^{56}$.

55. C'est le cas de Dilsey et de Luster qui prononcent de façon répétée «Miss Ca'oline»; du Noir entrevu de la fenêtre du train «Me'ci, mon jeune maître. Me'ci bien.» (Pléiade:424); du Deacon «Pa'ici mon jeune maître” (Pléiade:433); du pompiste qui s'adresse à Jason «Pa'tez en voyage» (Pléiade:617); puis des deux jeunes garçons qui donnent leur prix pour s'occuper de la voiture de ce dernier: «Quatre dolla'.» (Pléiade:62) Le fait n'est donc pas isolé. Il aurait par contre tendance à s'attacher à des personnages transitoires, entrevus l'espace d'un instant.

56. Bakhtine, op. cit., 1978, p. 152. En fait, nous voudrions ici citer Bakhtine de façon plus complète, sa remarque nous semblant particulièrement appropriée. «Le romancier ne connait pas de langage seul et unique, naïvement (ou conventionnellement) inconstestable et péremptoire. Il le reçoit déjà stratifié, subdivisé en langages divers. C'est pourquoi, même si le plurilinguisme reste à l'extérieur du roman, même si l'auteur se présente avec un seul langage, totalement fixé (sans distanciation, ni réfraction, ni réserves), il sait que ce langage n'est pas signifiant pour tous ou incontestable, qu'il résonne au milieu du plurilinguisme, qu'il doit être sauvegardé, purifié, défendu, motivé. Aussi, même ce langage-là, unique et direct, est polémique et apologétique, autrement dit, dialogiquement corrélaté au plurilinguisme. C'est ce qui détermine la visée tout à fait spéciale, contestée, contestable et contestant, du discours romanesque: il ne 
Certes, le cliché est difficilement contournable et la Dilsey anglaise égrenne dans son discours un certain nombre de procédés reconnus, formant un noyau dur de stéréotypes - qui migrent d'ailleurs d'un personnage à l'autre et d'un récit à l'autre. Toutefois, sa négritude ne se synthétise pas non plus dans une sonorité unique : s'y ajoutent de nombreux marqueurs idiolectaux. Elle prononce tantôt «Ca'oline» tantôt «Cahline»; son accent est plus ou moins prononcé selon les circonstances, son interlocuteur ou son âge; certains particularismes phonétiques lui appartiennent en propre, etc. Aussi ne pouvons-nous abusivement reprocher au traducteur de s'appuyer en langue d'arrivée sur un noyau équivalent. N'oublions pas en outre que le stéréotype, aussi poisseux soit-il, a néanmoins un avantage sur le point de vue de l'éviction pure et simple: il reconnaît dans l'acte même du jugement qu'il porte, l'altérité de l'autre, son existence. Ce n'est donc pas sur la stérétypification en elle-même que portent nos objections, contrairement à la position de $\mathbf{M}$. Gresset, mais sur le fait que le processus de réintégration se limite étroitement à l'élision du «r». Procédé qui participe plus du banal que de la créativité d'une part, et qui donne à penser d'autre part que l'univers de Coindreau ne peut admettre la négritude du personnage qu'en autant qu'elle passe par le laminoir du cliché.

En fait, cette dernière remarque semble être corroborée par l'attitude du texte d'arrivée à l'égard de tous les accents, qu'ils figurent dans Le Bruit et la fureur ou Manhattan Transfer. Elle constitue même la condition sine qua non de l'acceptabilité. En effet, le traducteur, soucieux de l'oreille réceptrice, ne lui livre que des accents dont les sonorites sont familières. Accents qui ont depuis longtemps acquis droit de cité dans l'horizon d'attente de la société française; dont la fréquence permet une saisie facile et une lecture tranquille. De sorte qu'un Italien ou un Allemand garderont aisément en français les inflexions de leur langue d'origine. Il faut dire que dans l'un et l'autre cas la représentation artistique - des opéras bouffes de Rossini aux vaudevilles boulevardiers du tournant du siècle en passant par les romans populistes anti-germaniques - avait dûment préparé le terrain. Aussi, lorsque dans la deuxième partie de The Sound and the Fury

peut, ni naïvement, ni de manière convenue, oublier ou ignorer les langues multiples qui l'environnent.» 
(June second 1910), survient un jeune immigrant italien, le Bruit et la fureur n'a aucun mal à s'italianiser à l'unisson: Julio, convaincu d'avoir affaire au ravisseur de sa jeune sœur Lisa, lance à Quentin une série d'injures et de menaces dans un anglais approximatif, mais dont les inflexions sont immédiatement identifiables ${ }^{37}$.

"I killa heem," Julio said.

"You steala my seester," Julio said. "Let go, meesters." (Vintage: 174)

"Git on home," Julio shouted at her, "I beat hell outa you." (Vintage: 177)

"I American," Julio said. "I gotta da pape'." (Vintage:179)

"Sure I quit. I run. I run like hell. Looka here, looka there, then man tella me he seen him giva her she eat. She go weetha." (Vintage:179)

La traduction de Coindreau, conciliante, conserve aux propos du jeune homme toute la saveur de son pays d'origine. Que l'on en juge:

- Io lé tourai», dit Julio.

«Vous avez volé ma sœur, dit Julio. Lâchez-moi, signore. (Pléiade:471).

«Rentre à la maison, lui hurla Julio. Io té foutrai oune raclée.» (Pléiade:473)

- Moi Américain, dit Julio. Io avé tutti mi papiers. (Pléiade:474)

57. Si nous élargissons notre observation à la première oeuvre de Coindreau traduite de l'américain, Manhattan Transfer, oeuvre polylingue à l'excès, la tendance se confirme puisque l'un des rares accents à recevoir une représentation intégrale est celui de l'allemand, Mr. Zucker, au tout début du roman. Là encore, le discours populaire fournissait un arsenal éprouvé. Que l'on en juge:

«Foulez-fous me permettre, monsieur, de vous offrir à poire pour fêter l'éfénement? [...] Ach!... la fie de famille tonne pien des soucis. [...] Foulez-vous te la pière? [...] Teux pouteilles de Culmbacher importée, pour poire à notre petite famille.» (Folio, pp. 15-17) 
- Sour que io quitté. Io couru, couru como l'inferno. Io regardé ici, io regardé là, pouis un uomo il m'a dit qu'il l'avait vou lui donner à manger. Elle allé avec loui.» (Pléiade:475).

En somme, pour nous résumer, le parti-pris de Coindreau face au vernaculaire noir consiste en une attitude à double volet, apparemment sereine dans sa théorisation, en réalité contradictoire dans son application ${ }^{38}$. D'une part, il proclame l'impossibilité d'une restitution et préconise l'éviction; de l'autre, il la réintroduit subrepticement et se replie pour ce faire sur le procédé que lui-même anathématisait. En outre, le rejet systématique et maintes fois répété, dont il fait montre à l'égard de l'accent noir ne s'applique pas uniformément, ni avec la même rigueur, envers les autres accents, comme nous l'avons vu. Si un Italien ou un Allemand conservent les inflexions de leur langue d'origine, les Noirs, eux, succombent au classicisme bourgeois de leur interprète. Toutefois, gardons-nous de conclure hâtivement à un parti-pris ségrégationniste déguisé: le traducteur s'en tient scrupuleusement à ce que lui permettait sa société, quitte à contredire sa propre thérie.

58. Il existe d'autres exemples de contradiction entre la théorie et la pratique de Coindreau. Ainsi de la citation suivante: «The reasons for the popularity and impact of a foreign literary genre in a particular country at a particular time are always the same. The Foreign works act as a kind of blood transfusion. Either they reinforce already existing tendencies, or they bring new elements to genres beginning to show signs of pernicious anemia.» (p. 19; c'est nous qui soulignons.) Cet argument ne serait pas sans évoquer la position de Meschonnic et de Berman lorsqu'ils préconisent la métisation de la langue d'arrivée, ou le décentrement. Ce que Coindreau n'a pas su mettre en pratique à propos du parler noir. Il s'en est même fortement défendu: «Any translator who knows to write will find no difficulty in reproducing these «moments, characters, rhythms of speech, and if he knows his mother tongue well, he will know how to make a peasant or a poet speak convincingly. If he were to forget the principles so intelligently set forth by William Faulkner, he would nun the risk of making a colored woman from Mississippi talk like a Negress from Martinique, Guadeloupe, or Haiti.» The Time of William Faulkner, pp. 89-90. 
La résistance d'un langage canonique, seul et unique, étayé par l'unité encore inébranlable du mythe national, est trop forte encore pour que le multilinguisme puisse relativiser et décentraliser la conscience littérairement linguistique. Cette décentralisation verbale et idéologique ne se fera que lorsque la culture nationale aura perdu son caractère clos, autonome, quand elle aura pris conscience d'elle-même parmi les autres cultures et langues ${ }^{59}$.

\section{Conclusion}

Pour terminer, il nous semble important de souligner que ces divers procédés de blanchiment ${ }^{60}$, déjà difficilement acceptables en eux-mêmes, deviennent franchement insupportables lorsqu'ils sont placés en regard de l'Histoire des Noirs aux États-Unis. Histoire qui nous a appris de façon répétée que, d'abord spoliés de leur liberté par l'esclavage, ensuite de leurs droits civiques par la ségrégation, puis de leur musique par le rock and roll, les Noirs le furent aussi de leur langage par la linguistique étasunienne. N'oublions pas que ce n'est que depuis les années 60 que les linguistes ont accepté de prendre en compte le substrat africain du vemaculaire noir. Auparavant l'origine du Black English ne se concevait avant tout qu'en termes de l'influence anglo-saxonne. Tout remontait à l'Angleterre, et au May Flower. Bref, les Noirs ne pouvaient pas même prétendre à la paternité de leur langue.

American linguistics has failed to provide accurate information about Black English because of the myopic nature of most

59. Bakhtine, op. cit. 1978, p. 186.

60. Nous hésitons à parler de blanchissement du fait que ce terme recouvre une réalité bien précise dans la traite des Noirs. Après que le capitaine du navire négrier avait subi la quarantaine de rigueur, et qu'il s'apprêtait à vendre sa cargaison humaine, il soignait et "remettait en état» sa marchandise quelque peu défraîchie après la longue traversée. Meilleure nourriture, coupe des cheveux, maquillage des défauts physiques, c'est ce que l'on appelait alors le blanchissement. 
historical work on American English. Dialectologists, the researchers to whom most such investigations have been entrusted, have concerned themselves almost exclusively with patterns of migration from the British Isles and with what they think of as the spread of British regional features throughout the United States ${ }^{61}$.

Le contexte des années 90 ne permet plus l'aphasie ni la cécité ayant prévalu jusqu'à maintenant. L'excuse d'un absolu de beauté et de «bon goût», notions nécessairement idéologiques donc variables ${ }^{62}$, ne saurait justifier l'éviction du vernaculaire noir des textes français. Tout défaut de reconnaître ces simples vérités équivaut, à nos yeux, à une injustice égale à celle dont les Noirs ont eu à souffrir dans leur propre réalité.

61. J.L. Dillard, op. cit. p. 3. «Attempts to trace the features of Black English to British regional dialects have been carried to absurb lengths. The third person singular, present tense of the verb without "s» («he do" rather than «he does») has been traced to East Anglia; the durative «be» («he be going every day rather than «he is going») to Anglo-Irish; and preverbal «done» (he done go, he done gone, he done went») to Scottish dialect. For a population group with no history of residence in the British Isles, this is a wide collection of British features. Furthermore, how did features from such diverse areas of the British Isles come into the English of American Blacks - particularly since Scottish, Irish and East Anglian immigrants to the United States did not transmit those features to their descendants? A more important criticism of the geographers' position is that the grammar of Black English does not match the grammar of the allegedly identical features in British regional dialects.» (p. 7, pour ce dernier extrait)

62. Cette variabilité devient particulièrement irritante quand on compare les traductions possibles du vemaculaire noir. L'ouvrage récent d'Alice Walker, The Color Purple, ne parvient pas dans sa traduction française à recréer l'atmosphère verbale du texte anglais. Par contre, une nouvelle édition de Huit hommes de Richard Wright aux Éditions Gallimard accepte la négritude (élision du «r») des personnages. 
Pour ce faire, un changement d'attitude devient nécessaire et urgent. Changement à l'égard du VNA, certes, mais aussi et avant tout du dialogue romanesque, ou, si l'on préfere, de façon élargie, de la mimésis verbale. Il s'agit ni plus ni moins que de réattribuer à celle-ci une place plus juste dans les études littéraires en premier lieu, puis de se sensibiliser à une nouvelle éthique du traduire face au parlé. Nous avons là «la quintessence des problèmes que pose la traduction romanesque - le problème exigeant une réflexion maximale du traducteur ${ }^{63}$. La conscience bakhtinienne du langage peut nous y aider, nous l'avons vu : dans la multiplicité de ses appréhensions (dialogisme, plurilinguisme, polyphonie, hétérologie, hétéroglossie), elle favorise une prise en compte du foisonnement et de la superposition des langages, qui ne peut qu'enrichir les études en traductologie.

Comment restituer des textes enracinés dans la culture orale dans une langue comme la nôtre, qui a suivi une trajectoire historique, culturelle et littéraire inverse [de l'allemande]? On pourrait ne voir là qu'un problème technique, sectoriel, et c'est tout. Mais en vérité, il y a là un défi qui met en jeu le sens et le pouvoir de la traduction. Le travail à accomplir sur le français moderne pour le rendre capable d'accueillir authentiquement, c'est-à-dire sans ethnocentrisme, ce domaine littéraire montre bien qu'il s'agit, dans et par la traduction, de participer à ce mouvement de décentrement et de changement dont notre littérature (notre culture) a besoin si elle veut retrouver une figure et une expérience d'elle-même qu'elle a en partie perdues (pas totalement, bien sûr!) depuis le Classicisme $^{64}$.

GRETI*, Université McGill

- Le Groupe de recherche en traductologie (GRETI) est subventionné par le Conseil de recherches en sciences humaines du Canada.

63. Antoine Berman, op. cit. (1985), p. 79.

64. Antoine Berman. L'Épreuve de l'étranger. Culture et traduction dans l'Allemagne romantique (Paris, Gallimard, 1984), pp. 38-39. 